$$
\text { GYPSY FIELD PROJECT }
$$$$
\text { IN RESERVOIR CHARACTERIZATION }
$$$$
\text { DOE/BC/14869--2 }
$$

Contract Number:

Contractor:

Contract Date:

Anticipated Completion Date:

Government Award

Principal Investigator:

Project Manager

Reporting Period

Submitted to:
DE-FG22-95BC14869

University of Oklahoma

Center for Reservoir Characterization

Norman, Oklahoma 73019

April 6, 1995

June 30, 1996

$\$ 350,000$ (Current Year)

Daniel J. O’Meara Jr.

Robert E. Lemmon

Bartlesville Project Office

July 1 - September 30, 1995

Pittsburgh Energy Technology Center

U.S. Department of Energy

Wallace Road, Building 921 (Overnight Mail)

P.O. Box 10940, MS 921-118

Pittsburgh, Pennsylvania 15236

(412) 892-4862 (Telephone)

Document Control AD-20

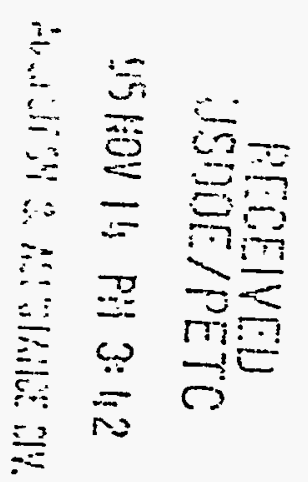

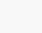

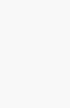




\section{GYPSY FIELD PROJECT IN RESERVOIR CHARACTERIZATION}

\section{Objectives}

The overall objective of this project is to use the extensive Gypsy Field laboratory and data set as a focus for developing and testing reservoir characterization methods that are targeted at improved recovery of conventional oil.

The Gypsy Field laboratory, as described by Doyle, O'Meara, and Witterholt (1992), consists of coupled outcrop and subsurface sites which have been characterized to a degree of detail not possible in a production operation. Data from these sites entail geological descriptions, core measurements, well logs, vertical seismic surveys, a 3D seismic survey, crosswell seismic surveys, and pressure transient well tests.

The overall project consists of four interdisciplinary sub-projects which are closely interlinked:

1. Modeling depositional environments.

2. Upscaling.

3. Sweep efficiency.

4. Tracer testing.

The first of these aims at improving our ability to model complex depositional environments which trap movable oil. The second entails testing the usefulness of current methods for upscaling from complex geological models to models which are more tractable for standard reservoir simulators. The third investigates the usefulness of numerical techniques for identifying unswept oil through rapid calculation of sweep efficiency in large reservoir models. The fourth explores what can be learned from tracer tests in complex depositional environments, particularly those which are fluvial dominated.

\section{Summary of Technical Progress}

During this quarter, the main activities involved the "Modeling depositional environments" Project", for which the progress is reported below: 
We seek to quantify the resolution of properties of an estimated parameter, cf [8]. In particular we are interested in whether features can be resolved or at least detected from a set of data with a given estimation procedure for a given model. The related notion of the sensitivity with respect to perturbations of the data has received much attention in the literature $[1,4,5,6,10,11]$. Here we consider sensitivity with respect to data for interior optimal estimators as well. In addition, however, we wish to view the manner in which validating numerical experiments for estimation problems are formulated. One approach to validation specifies a coefficient that is used in a model set of equations to generate data by solving the so-called forward problem. That data (with perhaps some added noise) is then used in the estimation algorithm to attempt to recover the original coefficient. The comparison of the specified test coefficient and the estimated coefficient is examined to determine the performance of the procedure. Since there may be multiple solutions, the mapping taking the data to the optimal estimator may not be well-defined as a function but as only a set-valued mapping. Nevertheless, it is possible to give sufficient conditions for the uniqueness or local uniqueness of optimal estimators and thereby define a function. In fact this mapping, which we refer to as the recovery mapping, is defined from the test parameter space to the space in which the estimated parameter resides. Clearly, the recovery mapping is the composition of the parameter-to-state mapping, the state-to-data mapping, and the data-to-estimated parameter mapping. Thus, it depends on the model, the data, the estimation method, and the approximation procedure. Our view of the study of the resolution of a procedure amounts to the study of the properties of the derivative of the recovery mapping.

Of course in an application, one may not have sufficient information available to determine how well the method has detected features. However, with the availability of a mathematical model, it is possible to analyze this dependence within the context of the model. Consideration of such properties would appear to be useful in the design of, experiments in order to extract information for systems to which the model applies. For example, one could examine the best location for observers in order to optimize sensitivity to perturbations of the estimator in some sense. Also, one could consider the value of new data. That is, how much does the introduction of new data add to the knowledge of the estimated coefficient. Further, it is sometimes important simply to know whether a method is capable of detecting a feature in order to apply more specialized techniques that may take advantage of other information [10].

We restrict our attention to finite dimensional systems arising from finite element approximations of elliptic boundary value problem and to optimal estimators in the interior of the set of admissible parameters. The results we obtain rely on the calculation of the rank and the eigenvalues/eigenvectors of certain matrices. From these we construct what we call a sensitivity matrix that allows us to consider the effect perturbations from certain subspaces have on the estimated parameters. Note that these are algebraic considerations. Because of roundoff error, rarely does one actually have a calculation resulting in zero as required in determining a null space. Nevertheless, one can obtain rank information from most computational software packages. One could perhaps consider the effective null 
space of the sensitivity matrix corresponding to eigenspaces associated with suitably small eigenvalues. These issues will be explored in later work.

The contribution of this work is to analyze within the context of interior optimal estimators the sensitivity of the recovery function to perturbations for finite dimensional estimation problems. If one relates the perturbation of the parameter vector to perturbations of the parameter function in the distributed system, then one is led to an approach for using the mathematical model to analyze the sensitivity of estimators to perturbations of the model coefficients. These methods may be used to optimize the problems. For example, we indicate an algorithm to locate observers according to a criteria that combines the fit-to-data functional with derivative information. It is the hope that by conducting such analyses one can design better experiments to evaluate information available and to assess the value of additional information perhaps to reduce the cost of expensive data collection.

We illustrate our ideas with a simple example. Suppose that $\Omega$ is the open unit interval' $(0,1)$. It is desired to estimate the coefficient $a=a(x)$ in the boundary value problem

$$
\begin{aligned}
& -\left(a u_{x}\right)_{x}=f \text { in } \Omega \\
& u(0)=u(1)=0
\end{aligned}
$$

from measurements of $a$ and $u$ at points in $(0,1)$. To discribe the test problem further, let $(0,1)$ be partitioned into $2 \mathrm{M}$ subintervals of equal length. Let $\mathrm{N}=2 \mathrm{M}-1$ and let $\left\{B_{k}\right\}_{k=1}^{N}$ be a set of piecewise linear basis functions with boundary conditions $B_{k}(0)=B_{k}(1)=0$. Also, we introduce the functions

$$
b_{k}(x)=\left\{\begin{array}{l}
1 \text { if } \mathrm{x} \in\left(\frac{(k-1)}{M}, \frac{k}{M}\right) \\
0 \text { otherwise }
\end{array}\right.
$$

for $k=1, \ldots, M$. For the purposes of our discussion we take $M=4$. Further, to simplify matters the functions $\left\{b_{k}\right\}_{k=1}^{M}$ will be used as a set of basis functions to express both the test parameter and the parameter to be estimated. Hence, the test coefficient $\alpha$ is expressed as

$$
\alpha=\sum_{k=1}^{M} \alpha_{k} b_{k} .
$$

We use $\alpha$ to denote the vector

$$
\alpha=\left[\begin{array}{c}
\alpha_{1} \\
\cdot \\
\cdot \\
\cdot \\
\alpha_{M}
\end{array}\right]
$$

Define the matrices $G^{(k)}$ for $\mathrm{k}=1, \ldots, \mathrm{M}$ by

$$
G_{i j}^{(k)}=\int b_{k} \nabla B_{i} \cdot \nabla B_{j} d x
$$


and the stiffness matrix $\Gamma$ by

$$
\Gamma=\sum_{k=1}^{M} \alpha_{k} G^{(k)}
$$

Given the forcing vector

$$
F=\left[\begin{array}{l}
0 \\
1 \\
0 \\
0 \\
0 \\
0 \\
0 \\
0
\end{array}\right],
$$

we solve the equation

$$
\Gamma \mathrm{z}=F
$$

to find function

$$
z(x)=\sum_{k=1}^{N} z_{k} B_{k}(x)
$$

where

$$
\mathbf{z}=\left[\begin{array}{c}
z_{1} \\
\cdot \\
\cdot \\
\cdot \\
z_{N}
\end{array}\right]
$$

Taking the observation functionals $\Delta_{i}=\delta_{x_{0 i}}$ for $i=1, \ldots, N_{o}$ and $\Theta_{i}=\delta_{x_{p i}}$ for $i=1, \ldots, N_{1}$ where $\delta_{x}$ denotes the Dirac delta measure with mass at $x$, we define $\Phi$ as the $N_{o} \times M$ matrix given by

$$
\Phi=\left[\begin{array}{c}
B_{1}\left(x_{s 1}\right) B_{2}\left(x_{s 1}\right) \ldots B_{M}\left(x_{s 1}\right) \\
\ldots \\
B_{1}\left(x_{s N_{o}}\right) B_{2}\left(x_{s N_{o}}\right) \ldots B_{M}\left(x_{s N_{o}}\right)
\end{array}\right]
$$

with $\Phi_{2}=\Phi^{*} \Phi$. Data on the state is given as an $N_{o}$ - vector in terms of a product

$\Phi \mathbf{z}$

The matrix $\Psi$ is the $N_{1} \times N$ matrix given by

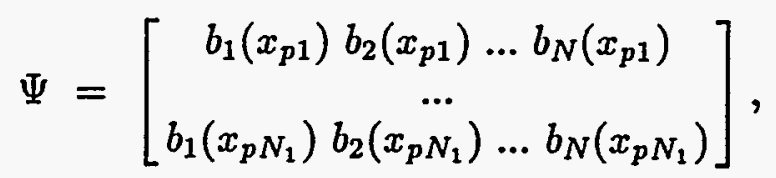


with $\Psi_{2}=\Psi^{*} \Psi$. Parameter data are given as an $N_{1}$ - vector in terms of the product

$\Psi \mathbf{a}$.

We seek to estimate the function $a$ (or the vector a) using the basis functions $\left\{b_{k}\right\}_{k=1}^{N}$ in which $a$ is expressed as a sum

$$
a(x)=\sum_{k=1}^{N} a_{k} b_{k}(x),
$$

and the stiffness matrix is given by

$$
G=\sum_{k=1}^{N} a_{k} G^{(k)} .
$$

The solution function

$$
u(x)=\sum_{k=1}^{M} c_{k} B_{k}(x)
$$

associated with the vector $\mathbf{a}$ is obtained by solving the equation

$$
G c=F
$$

The finite dimensional version of the fit-to-data functional is given by

$$
J(a)=(c-z)^{*} \Phi_{2}(c-z)+a^{*}\left(H+\Psi_{2}\right) a-2 \alpha^{*} \Psi_{2} a+\alpha^{*} \Psi_{2} \alpha
$$

where we take $H$ to be such that $H+\Psi_{2}$ is positive definite. The unconstrained minimization problem

$$
\text { minimize } J(a) \text { subject to } a \in \mathbf{R}^{N}
$$

is solved by a steepest descent method to find a given $\alpha, \mathbf{z}$, and the observation location vectors $x_{s}$ and $x_{p}$. As an example, data is generated by solving the equation (1) where

$$
\alpha=[5,0.1,2,5]
$$

and is obtained from a direct measurement of $\alpha$ at $x_{p}=0.85$ and one measurement of $u$ at $x_{s}=0.2$. For the matrix $H$, we use

$$
H=\left[\begin{array}{llll}
1 & 0 & 0 & 0 \\
0 & 1 & 0 & 0 \\
0 & 0 & 1 & 0 \\
0 & 0 & 0 & 0
\end{array}\right]
$$


so that $H+\Psi_{2}$ is positive definite. The vector $\mathbf{a}$ is estimated to be

$$
\mathbf{a}=[4.6,0.11,1.6,5]
$$

To determine the "best" location of the observer, we first look for a location such that the sensitivity matrix $\mathcal{S}=D \mathbf{a}(\alpha)$ has maximal rank. We then look for a location that is associated with a smallest minimal value of the functional $\mathrm{J}$ as compared with minimal values of $J$ at other locations while is some sense maximizing the norm of the derivative. In Figure 1 the graph of the minimal values of the fit-to-data functional $\mathrm{J}$ versus the observation point is given note that the minimal value occurs for the observation located at $x_{s}=0.9$. However, we look for a location that minimizes

$$
J k=J+0.0001 /\|S\| \text {. }
$$

The differentiability properties have been noted in the previous section. With the small size of the problem, however, we simply check the value of $J k$ at various points

$$
x_{s}=i * 0.1 \text { for } i=1, \ldots, 9 \text {. }
$$

For each point $x_{s}$, we can obtain the rank of the associated sensitivity matrix. The resulting numbers are arranged as a vector

$$
r=[2,2,2,2,2,2,2,2,1]
$$

each entry of which is the rank. Note that $x_{s}=0.9$ yields a rank of one so we look for an observation location $x 0(i)=i * 0.1$ for $i=1, \ldots, 8$. The graph of the associated $J k$ is given in Figure 2. Accordingly, we situate the observer at $x_{s}(1)=0.4$

To locate a second observation point, we continue in order to determine $x_{s}(2)$ where $x_{s}(1)=0.4$. In this case we find the rank vector

$$
r=[3,3,3,2,3,3,3,3,2] \text {. }
$$

In Figure 3 the graph of $J k$ is presented. We see that $x_{s}(2)=0.8$ should be chosen. Finally, to select the point $x_{s}(3)$ we observe that the rank vector

$$
r=[4,4,4,3,3,3,3,3,3]
$$

Graphing $J k$ in Figure 4, we see that the point $x 0(3)=0.1$ yields the minimimal value of ' $J k$ over those location that are associated with rank 4 even though there are locations at. which the value of $J k$ is smaller.

Observe that the procedure seeks more than the location yielding the minimum value of J. Rather the attempt is to maximize the effect of the derivative as well by determining the minimum of $J k$ over those locations with maximum rank. 
Finally, we briefly present an example to observe directly the effect perturbations of $\alpha$ have on the estimate $\mathrm{a}(\alpha)$. To do this, we begin with the vector $\alpha=[5,0.1,2,5]$ and consider the estimated value obtained as two of the three components are fixed while the remaining is allowed to vary as an initial guess in the minimization procedure. Suppose the observation point is

$$
x_{s}=0.4 \text {. }
$$

In this case varying the first component only as an initial guess, we obtain

$$
\mathrm{a}(\alpha)=[3.9,0.093,2,5] \text {. }
$$

Perturbing $\alpha$ by $\alpha^{\prime}= \pm[0.1,0,0,0]$, we find

$$
\mathrm{a}(\alpha+[0.1,0,0,0])=[3.9,0.093,2,5] \text { and } \mathbf{a}(\alpha+[-0.1,0,0,0])=[3.9,0.094,2,5] \text {. }
$$

Next, fixing the first and third components and varying only the second component in the initialization of the minimization procedure, we have

$$
\mathrm{a}(\alpha)=[5,0.09,2,5] \text {. }
$$

Perturbing $\alpha$ by $\alpha^{\prime}= \pm[0,0.01,0,0]$, we obtain

$$
\mathrm{a}(\alpha+[0,0.01,0,0])=[5,0.1,2,5] \text { and } \mathbf{a}(\alpha+[0,-0.01,0,0])=[5,0.08,2,5] .
$$

Lastly, fixing the first and second components while varying the only the third in the initialization of the minimization procedure, we find

$$
\mathrm{a}(\alpha)=[5,0.095,0.7,5] \text {. }
$$

Perturbing $\alpha$ by $\alpha^{\prime}=[0,0,0.1,0]$, we obtain

$$
\mathrm{a}(\alpha+[0,0,0.1,0])=[5,0.095,0.7,5] \text { and } \mathbf{a}(\alpha+[0,0,-0.1,0])=[5,0.095,0.7,5] \text {. }
$$

On the otherhand, suppose the observation point is

$$
x_{s}=0.8 \text {. }
$$

As in the previous case varying the first component only as an initial guess, we obtain

$$
\mathrm{a}(\alpha)=[0.91,0.094,2,5] \text {. }
$$

Perturbing $\alpha$ by $\alpha^{\prime}= \pm[0.5,0,0,0]$, we find that

$$
\mathrm{a}(\alpha+[0.5,0,0,0])=[0.91,0.094,2,5] \text { and } \mathbf{a}(\alpha+[-0.5,0,0,0])=[0.91,0.094,2,5]
$$

Next, fixing the first and third components and varying only the second component in the initialization of the minimization procedure, we find

$$
\mathrm{a}(\alpha)=[5,0.08,2,5] \text {. }
$$


Perturbing $\alpha$ by $\alpha^{\prime}= \pm[0,0.01,0,0]$, we get

$$
\mathrm{a}(\alpha+[0,0.01,0,0])=[5,0.09,2,5] \text { and } \mathrm{a}(\alpha+[0,-0.01,0,0])=[5,0.07,2,5] \text {. }
$$

Lastly fixing the first and second components while varying the only the third in the initialization of the minimization procedure, we have

$$
\mathrm{a}(\alpha)=[5,0.1,0.11,5] .
$$

Perturbing $\alpha$ by $\alpha^{\prime}= \pm[0,0,0,0.1]$, we obtain

$$
\mathbf{a}(\alpha+[0,0,0.1,0])=[5,0.1,0.11,5] \text { and } \mathbf{a}(\alpha+[0,0,-0.1,0])=[5,0.1,0.11,5] \text {. }
$$

The point $x_{s}=0.4$ is an optimal location for an observation point in the sense that the rank of the sensitivity matrix $\mathcal{S}$ is maximized while and the functional $J k$ is minimized with respect to the set of locations tested, see Figure 2. Both $x_{s}=0.4$ and 0.8 are locations at which the respective sensitivity matrices have rank 2 . Indeed, both observers appear to be sensitive to one form of the perturbations. However, the norm of the derivative is larger for the case in which the observation point is 0.4. Indeed, for a perturbation of $\alpha^{\prime}= \pm[0.5,0,0,0]$ such as that used for the case with observation point 0.8 , we obtain ."

$$
\mathbf{a}(\alpha+[0.5,0,0,0])=[4.1,0.093,2,5] \text { and } \mathbf{a}(\alpha+[-0.5,0,0,0])=[3.7,0.094,2,5] \text {. }
$$

\section{DISCLAIMER}

This report was prepared as an account of work sponsored by an agency of the United States Government. Neither the United States Government nor any agency thereof, nor any of their employees, makes any warranty, express or implied, or assumes any legal liability or responsibility for the accuracy, completeness, or usefulness of any information, apparatus, product, or process disclosed, or represents that its use would not infringe privately owned rights. Reference herein to any specific commercial product, process, or service by trade name, trademark, manufacturer, or otherwise does not necessarily constitute or imply its endorsement, recommanufacturer, or otherwise does not necessarily constitute or imply its endors thereof. The views and opinions of authors expressed herein do not necessarily state or reflect those of the United States Government or any agency thereof. 


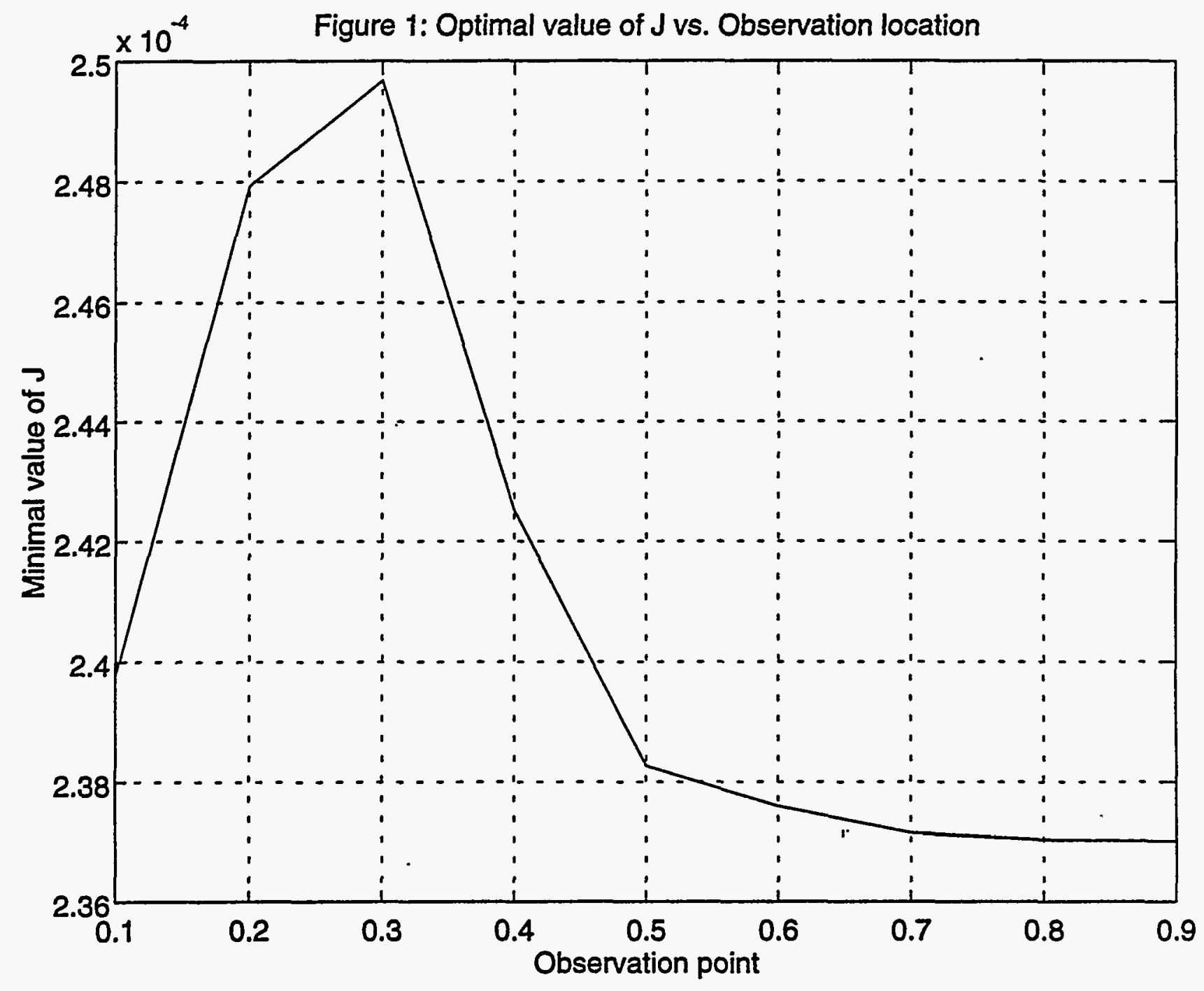




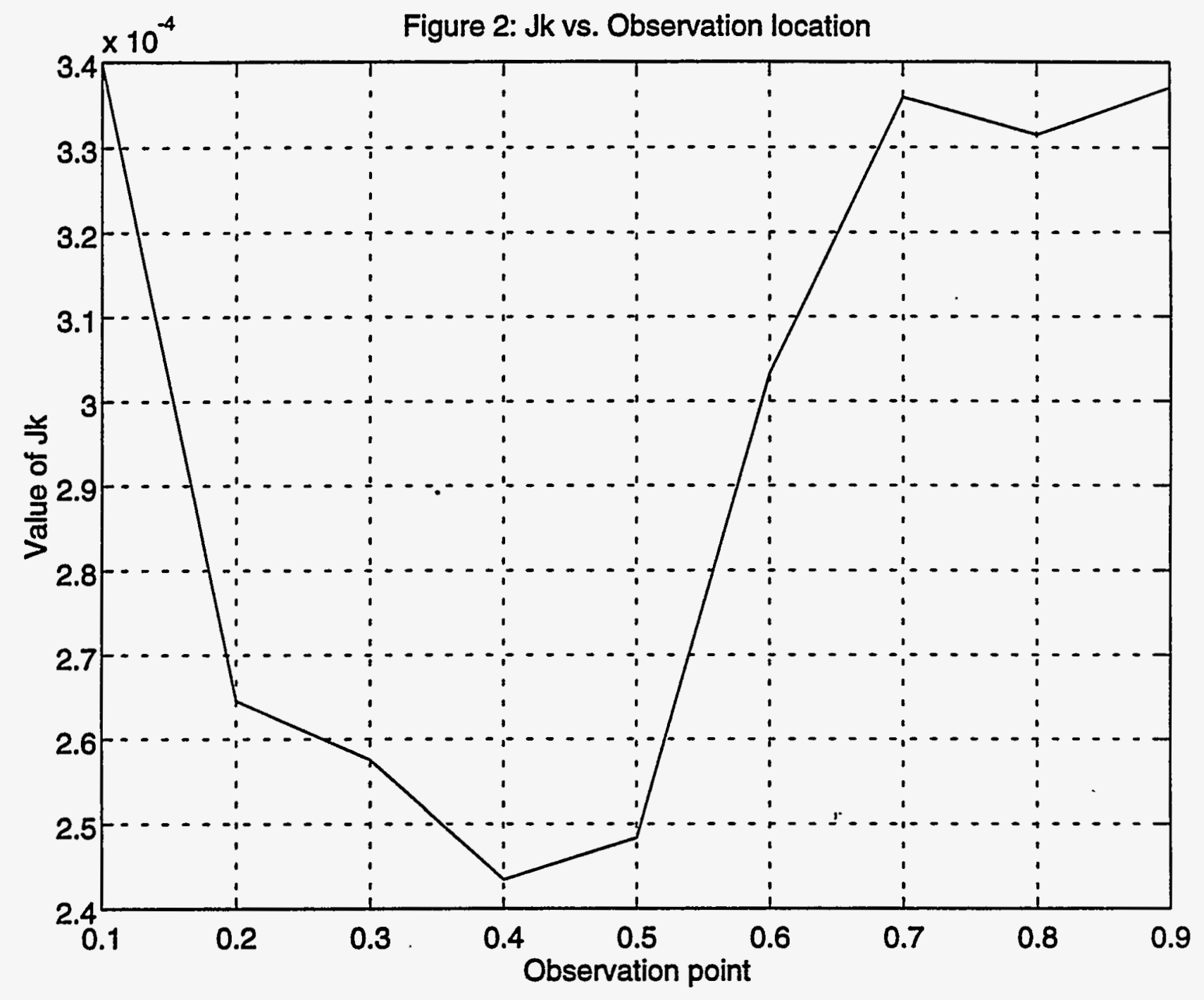




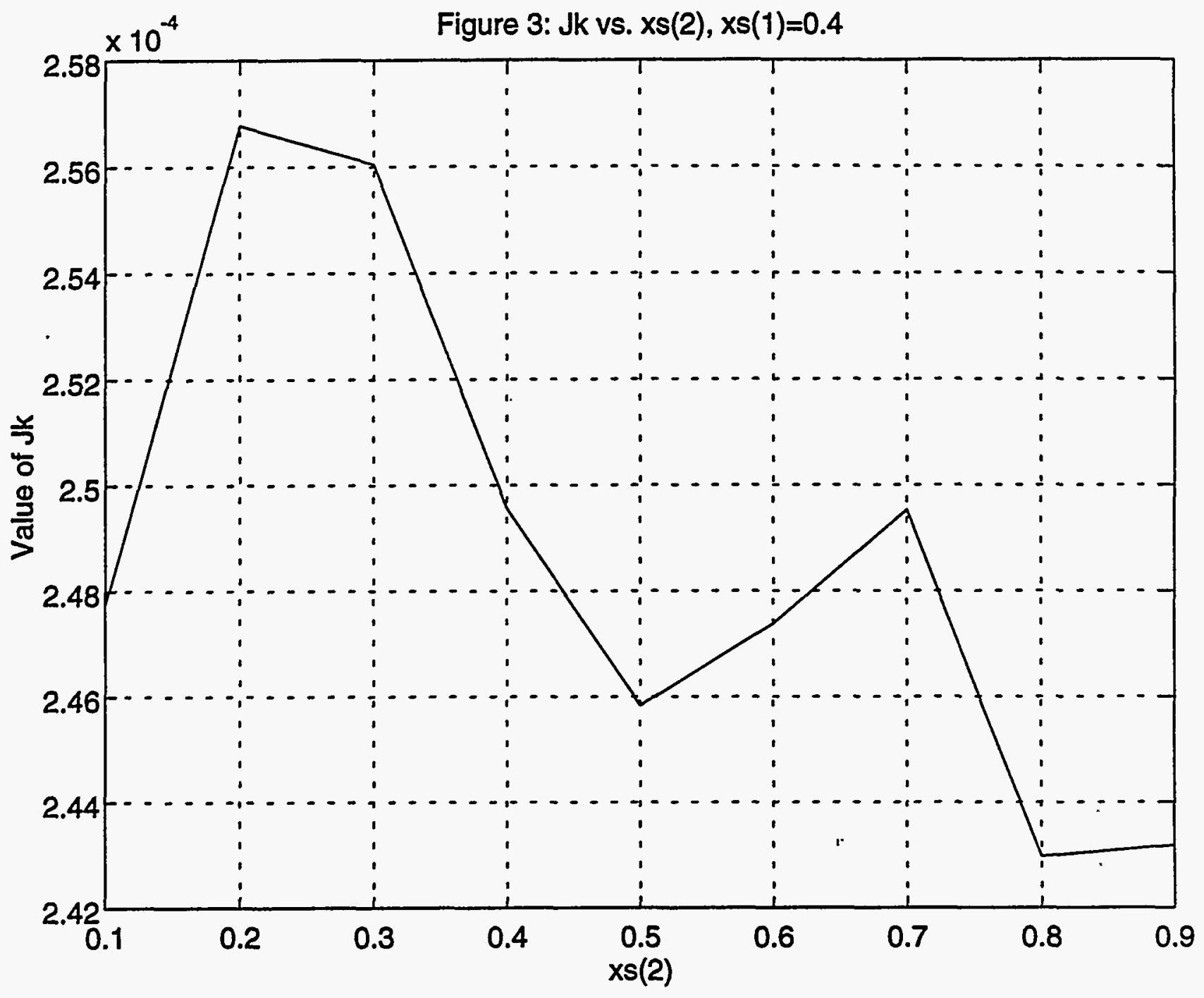




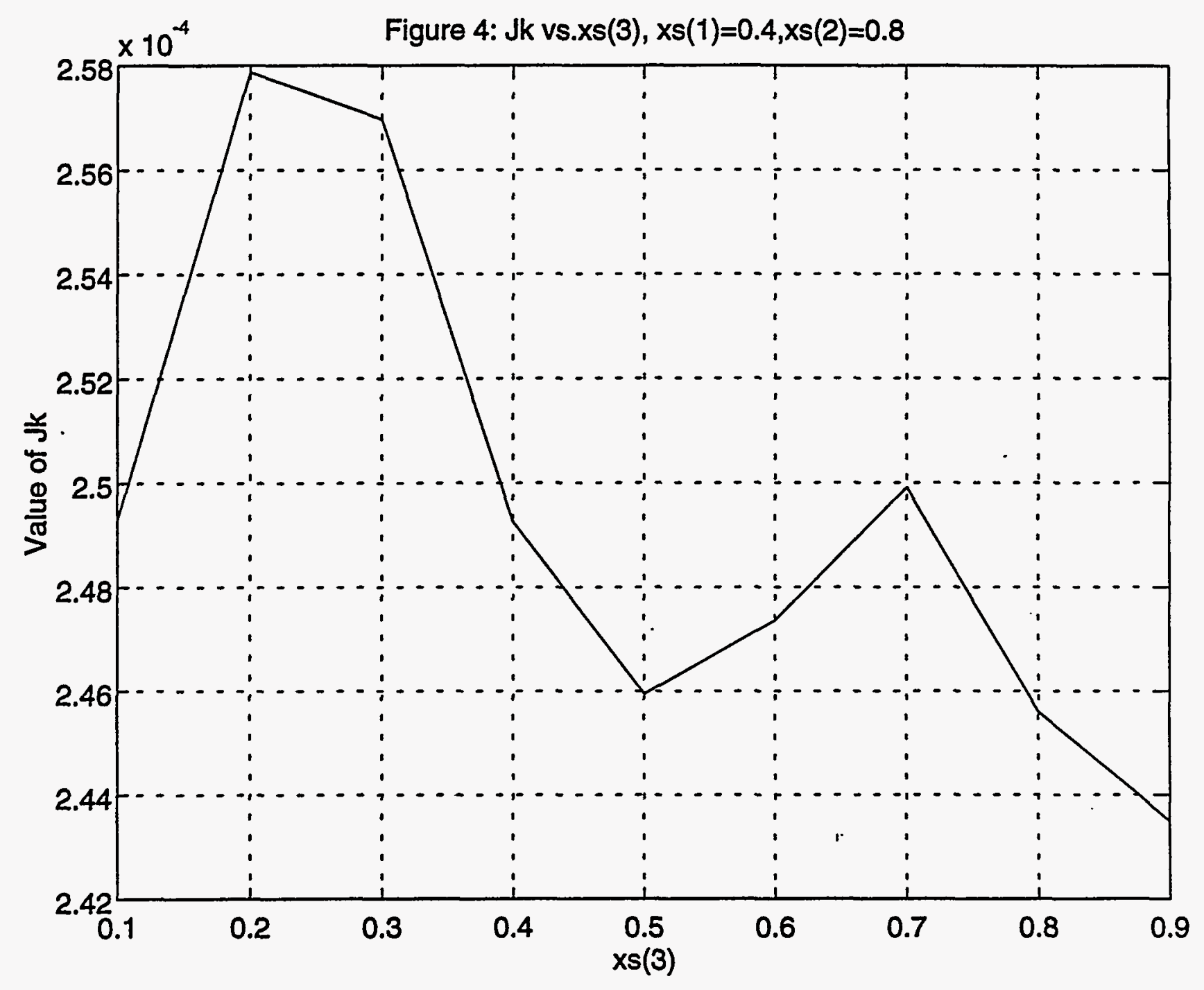




\section{REFERENCES}

[1] Colonius, F. and K. Kunisch, Output Least Square Stability in Elliptic Systems, Technische Universitat Graz, Report 86-76, June, 1986.

[2] Deimling, K. Nonlinear Functional Analysis, Springer-Verlag, New York, 1980.

[3] Kluge, R. An inverse problem for "coefficients" in linear equations. Uniqueness and iterative solution, Proceedings of the International Summer School in Nonlinear Analysis, Theory, and Applications, Akad-Verlag Berlin 1981, No. 1N, pp.71-88.

[4] Ito, K. and K. Kunisch, Maximizing Robustness in Nonlinear Illposed Inverse Problems, Siam. J. Control and Optimization, Vol. 33, No. 2, pp. 643-666, 1995.

[5] Ito, K. and K. Kunisch, On the injectivity of the coefficient-to-solution mapping for boundary value problems and its linearization, J.Math.Anal.Appl., to appear.

[6] Ito, K. and K. Kunisch, Sensitivity measures for estimation of parameters in elliptic boundary value problems, J. Math. Syst. Estimation and Control, to appear.

[7] Ladyzhenskaya, O.A. and N. Ural'Tseva, Linear and Quasilinear Elliptic Equations, Academic Press, New York, 1968.

[8] Parker, R.L. Geophysical Inverse Theory, Princeton University Press, Princeton,New Jersey.

[9] Schultz, M. Spline Analysis, Prentice Hall, Englewood Cliffs, New Jersey,1973.

[10] White, L.W., Y. Jin, and D.J. O'Meara, Jr., Estimation of Two Dimensional Reservoir Maps with Discontinuities, submitted.

[11] White, L.W. and J. Zhou, Continuity and Uniqueness of Regularized Output Least Squares Optimal Estimators, J. Math. Analysis and Applications, to appear. 